\title{
Protecting Intellectual Property Assets of Mushroom Genetic Resources for Invention and Innovation
}

\section{Shung-ChangJong}

American Type Culture Collection (ATCC), 10801 University Blvd., Manassas, VA 20110-2209, USA; sjong@atcc.org

The concept of intellectual property (IP) allows people to own creativity and innovations in the same way as physical property. An IP asset is any codified knowledge, innovation, or practice of actual or potential economic value, associated with fundamental research, analysis, and manipulation of biological systems, biological prospecting, industrial applications, or commercial use. Mushroom genetic resources are currently being utilized and exploited by the pharmaceutical, cosmetic, agricultural, food, enzyme, chemical, and waste-treatment industries. Nevertheless, the role of IP assets in today's knowledge-driven enterprises is frequently overlooked, despite their potential as sources of both monetary value and financial gain.IP rights are often under-managed or under-leveraged. The challenge is how to create, protect, and extract value from IP assets for invention and innovation.

In the valuation of IP assets, it is important to differentiate between "invention" and "innovation." Because an invention may not involve the commercialization of new ideas, it is obvious that not all inventions result in innovation. Innovation is an interactive process of effectively creating, managing, and leveraging an invention and successfully bringing new products to market. Therefore, IP assets can be used effectively to sustain mushroom production and business development.

The IP assets of mushroom genetic resources are divided into three basic yet interdependent components: (1) intellectual material, (2) intellectual capital, and (3) intellectual property. Intellectual material provides both tacit and codified knowledge of mushroom genetic materials including intangible content (genetic information), implemented know-how (product/process innovation), and knowledge databases (bioinformatics). Intellectual capital encompasses various kinds of knowledge and innovative ideas that are mainly driven by, and derived from, individuals or groups of scientists and technologists. Intellectual assets with legal protection become intellectual property. An IP right is thus a legal right granted or registered under the relevant national or regional law by the relevant IP office. Codified knowledge and know-how form a body of interactive IP assets that relates directly to product/process innovations and codified ideas of their creators and users.

It is often not possible to protect IP assets and gain IP rights unless they have been applied for and granted or registered through an IP system. IP assets that can be protected by formal legislation are patents, trademarks, copyrights, and plant breeders' rights. Know-how and trade secrets are forms of IP that are not protected by formal legislation but have national and international value. As with any property, IP assets possess specific dollar value and can be bought and sold.

One of the methods of gaining the value of mushroom genetic resources through IP is with a material transfer agreement (MTA). An MTA is a contract generally utilized when any proprietary 
material and/or information is exchanged with "trade secret" protection as embodied, for example, in various state laws in the United States. It defines the rights of the provider and the recipient with respect to the mushroom materials and any replicates or derivatives. A replicate is any mushroom material that represents a substantially unmodified copy of the original material and includes material produced by the growth of cells or amplification of material. A derivative is material created from the original material that is substantially modified to have new properties. Derivatives include recombinant DNA clones made using a vector. However, know-how and trade secrets are, in certain cases, protected contractually by the application of certain legal concepts and statutes, such as customary laws and practices. Signed contracts such as MTAs are the most powerful tools in trade secret and customary laws in which registration is not required.

Today, the new challenge for mushroom scientists and technologists is to realize that IP should no longer be considered as an inert legal title, but as an economical asset that can realize the potential of knowledge and creativity. PI assets are tools that maximize the value of special knowledge, either by providing data or information or by delivering expertise and augmentation to mushroom scientists and technologists. Concerns about access to the IP assets of mushroom genetic materials have recently surfaced because of the reluctance of IP owners to allow their use for research that may lead to commercial applications. IP holders need assurance that access will be controlled. A number of bilateral and multilateral initiatives have been implemented to protect IP assets among the proposed users. All agreements are negotiated in a manner that is coherent and mutually supportive of national and international laws, local customs, rules, and regulations and implemented through collaborative action by governments, appropriate organizations and professional societies, field collectors and their sponsors, and curators and users of mushroom genetic resources.

Although the Convention on Biological Diversity (CBD) establishes principles of access to, and benefit sharing of, biological materials, it does not establish any specific mechanisms for such activities. Several factors influence the design of mechanisms for access and benefit sharing. Two are of particular importance: (1) how clearly the target for access and sharing can be defined, and (2) whether the transaction costs associated with more precise targeting outweigh the benefits to be shared. At the same time, expectations of contingent returns must be tempered by the real probabilities of discovery of new applications. Different contractual arrangements have been designed for different resources and different uses. Negotiation of legal and policy tools for formulating and obtaining mutually agreed terms following prior informed consent can include many of the following: (1) access agreement, (2) confidentiality agreement, (3) material transfer agreement, (4) research agreement, (5) intellectual property protection agreement, (6) co-development agreement, (7) technology licensing agreement, (8) distributorship agreement, (9) commercial agreement, and (10) benefit-sharing agreement. 